\title{
Molecular Epidemiology and clinical Features Analysis of Respiratory Human Adenovirus Infections in Hospitalized Children: A Retrospective Study in Zhejiang
}

\section{Caiyun Wang ( $D 11281672 @ q q . c o m)$}

Zhejiang University School of Medicine Children's Hospital https://orcid.org/0000-0003-0932-3650

Juanjuan Liu

Zhejiang University School of Medicine Children's Hospital

Yumei Mi

Zhejiang University School of Medicine Children's Hospital Jing Chen

Zhejiang University School of Medicine Children's Hospital Jing Bi

Zhejiang University School of Medicine Children's Hospital Yinghu Chen

Zhejiang University School of Medicine Children's Hospital

\section{Research}

Keywords: Human adenovirus, Respiratory infection, Molecular epidemiology, Children

Posted Date: May 21st, 2021

DOl: https://doi.org/10.21203/rs.3.rs-521736/v1

License: (a) (i) This work is licensed under a Creative Commons Attribution 4.0 International License.

Read Full License 


\section{Abstract}

Background: HAdV is one of the common pathogens in hospitalized children with respiratory infection (RI). we aim to describe the epidemiological characteristics, clinical features, and genotype of inpatients with HAdV positive with RI.

Methods: Respiratory samples were gathered from inpatients diagnosed RI in Children's Hospital, Zhejiang University School of Medicine and were detected by using Direct Immunofluorescence Assay (DFA) from 2018 to 2019. PCR amplification and sequencing of the hypervariable zone of hexon gene were used for genotyping. The clinical and laboratory features, and typing of HAdV, and epidemiological characteristic analysis were retrospectively performed.

Results: Of 7072 samples collected, 488 cases were identified as HAdV-positive RI. The overall detection rate was $6.9 \%$. The peaked detection rate was $14.1 \%$ in January 2019 . HAdV-positive cases with RI mainly appeared in winter and summer. The detection rate was highest among children between 6 months and 2 years $(8.7 \%, 123 / 1408)$. Clinical diagnosis included pneumonia $(70.3 \%, 343 / 488)$, bronchitis $(7.0 \%, 34 / 488)$ and acute upper respiratory tract infection $(22.7 \%, 111 / 488)$. The common clinical manifestations were fever $(93.4 \%, 456 / 488)$, cough $(94.7 \%, 462 / 488)$, wheezing $(26.2 \%, 128 / 48)$, and shortness of breath (14.8\%, 72/488). $213(43.6 \%)$ cases had co-infection and $138(28.3 \%)$ cases had extrapulmonary symptoms. $96(19.7 \%)$ cases had intrapulmonary and intrathoracic complications.78 $(16.0 \%)$ had an underlying condition, the most of them were congenital heart diseases $(20.5 \%, 17 / 78)$. The proportions of hyperpyrexia, duration of fever more than 10 days, severe pneumonia, and proportion of wheezing in the co-infection group were remarkably higher than those in HAdV single-infection group $(p<0.05)$. 4 HAdV species were successfully identified in 155 cases and represented by 8 genotypes. HAdV-B3 $(56.1 \%, 87 / 155)$ and HAdV -B7 $(31.0 \%, 48 / 155)$ were the most predominant detected types and occurred commonly in different severity groups $(p=0.000)$, while, HAdV-B55 was detected only in the severe group. HAdV-B7's detection rate in the severe group was significantly higher than the non-severe infected group.

Conclusion: HAdV detection rate is related to age and season. Bronchopneumonia accounts for about $70 \%$ HAdV -positive inpatients. The common manifestations include hyperpyrexia, cough, wheezing, and shortness of breath. HAdV-B3 and HAdV-B7 are the most common types in children diagnosed with RI.

\section{Introduction}

Human adenovirus (HAdV) comprises A to $\mathrm{G}$ species which is subdivided into over 90 genotypes currently ${ }^{[1,2]}$. About $5-10 \%$ of all respiratory infections (RI) in infants and children are associated with HAdV ${ }^{[3,4]}$. HAdV was regarded as one of the frequent microorganisms attributed to $15 \%$ of ARI in hospitalized children ${ }^{[3,5,6]}$. A previous study revealed that clinical presentation of HAdV infection is mostly dependent on the genotype. HAdV genotypes associated with ARI include B (types 3, 7, 11, 14, 16, and 21), C (types 1, 2, 5, and 6) and E (type 4) ${ }^{[7]}$. Although HAdV infections cause light to moderate ARI 
within immunocompetent children, a serious condition may occur in younger children ${ }^{[1]}$, immunocompromised patients, and those with underlying chronic diseases which can cause significant morbidity and mortality ${ }^{[8,9]}$. HAdV often causes respiratory adenovirus epidemics and outbreaks in different provinces of China ${ }^{[10,11]}$, as well as in North America, Malaysia, and Korea ${ }^{[12-14]}$. The detection rate of HAdV in respiratory infection increased significantly recently.

It is necessary to strengthen surveillance of HAdV and observe its epidemic and infection characteristics. But so far now, a nationwide epidemiological surveillance program for HAdV infection has not yet been built in China. We intended to determine clinical and laboratory features, and typing of HAdV, and epidemiological characteristic in hospitalized children in Children's Hospital of Zhejiang University School of Medicine from August 2018 to September 2019.

\section{Materials And Methods}

\subsection{Study design, Case definition, and identification}

We conducted a retrospective review study. In this study, 7072 samples of the nasopharyngeal aspirates (NPAs) or nasal/nasopharyngeal swabs (in viral transport media) were gathered from hospitalized children under 18 years old with ARI at Children's Hospital of Zhejiang University School of Medicine in China between August 2018 and September 2019. Of these samples, 488 HAdV positive infections were identified as samples detected by D3 UItra ${ }^{\text {TM }}$ DFA Respiratory Virus Screening \& ID Kit (Diagnostic Hybrids, Inc., USA). Respiratory syncytial virus (RSV), human adenovirus, influenza A viruses (Flu A) and influenza B viruses (Flu B), and parainfluenza 1, 2, and 3 viruses were detected. All NPAs or nasopharyngeal swabs samples were collected within 24 hours after admission. The specimens were divided into two parts and preserved in standard transport media. Rapid antigen testing for specimens was performed based on the instruction of the manufacturer within 2 hours after collection. All the samples were stored immediately in a tube containing $1.5 \mathrm{ml}$ of viral transport medium at $-80^{\circ} \mathrm{C}$ for further analysis. Meanwhile, bronchial alveolar lavage fluids (BALF), pleural effusion (PE) (if available), and blood from HAdV-positive patients during hospital stay were used to identify other pathogens.

Patient age was defined according to the date of hospitalization. Case definition for RI was any cases with fever $\left(>37.5^{\circ} \mathrm{C}\right)$ at initial presentationखaccompanied by cough, expectoration, nasal obstruction, sneeze, sore throat, dyspnea during the previous week. All participants must satisfy the inclusion criteria as follows:

- age under18 years old and more than 28 days,

- disease course on admission $\leq 1$ week

- confirmed acute respiratory tract infection with fever at initial presentation, cough, nasal obstruction, expectoration, sneeze, dyspnea, tachypnea, dyspnea, or wheeling/rales upon auscultation.

- HAdV positive in the testing of NPAs or nasopharyngeal swabs samples through direct immunofluorescence assay. 
- provision of informed consent

The exclusion criteria were:

- disease course on admission more than one week

- infection occurred in the neonatal period

- acquired respiratory infection in hospital

- died or discharged within 48 hours after admission

- children with immunodeficiency including neurological diseases, inherited metabolic diseases, asplenia, cerebral palsy, and/or complex- conditions.

- cases with substantial missing data.

\subsection{Data collection}

Patient general demographics (including age, sex, and sample collection time), social, clinical characteristics, laboratory results, radiological findings, and disease severity of HAdV positive respiratory infection children admitted to our hospital were collected from the medical records after the patients' discharge from the hospital. Patient characteristics, symptoms, signs, diagnosis, and treatment were recorded. All the data were entered into a standard electronic database. Underlying medical diseases included congenital or acquired heart disease, asthma, epilepsy, liver, and gastrointestinal diseases, kidney disease, diabetes, or other endocrine diseases.

\subsection{Definition of clinical severity}

Patients were categorized into upper respiratory infection (URI) and lower respiratory infection (LRI) according to clinical diagnosis. Severe pneumonia was diagnosed according to American Thoracic Society's guideline for the management of community-acquired pneumonia ${ }^{[15]}$.

\subsection{Detection of HAdV and molecular genotyping}

Viral nucleic acids were extracted from specimens of the HAdV-positive NPAs or swabs using QIAamp MinElute Virus Spin Kit (QIAGEN, German) based on instructions of the manufacturer. Nested-PCR amplification that targeted highly variable regions of the hexon gene was proceeded to screen HAdV infection. The primers of nested-PCR were: PCR1-F 5'-GATGCCGCAGTGGKCKTACATG-3', PCR1-R 5'GCTTACAAYTCNCTSGCT-3', and PCR2-F 5'-GACGCYTCGGAGTACCTGAG-3', PCR2-R 5'-

GGCYAGCACNTACTTTGACATYCG -3'. The following reaction conditions were: initial denaturation at 94 ${ }^{\circ} \mathrm{C}$ for $10 \mathrm{~min}$ and then $35 \mathrm{cycles}$ of $1 \mathrm{~min}$ at $94^{\circ} \mathrm{C}, 1 \mathrm{~min}$ at $55^{\circ} \mathrm{C}$, and $2 \mathrm{~min}$ at $72{ }^{\circ} \mathrm{C}$, and an extension at $72{ }^{\circ} \mathrm{C}$ for $7 \mathrm{~min}$. PCR amplification products were purified by $1 \%$ agarose gel electrophoresis and then sent to Beijing Genomics Institute (BGI) for Sanger sequencing of hypervariable regions of hexon gene which were confirmed as true. Samples are defined as untyped which are unable to amplify. Sequencing results were sequenced on GenBank for typing. 


\subsection{Statistical Analysis}

The results were analyzed utilizing SPSS software (version 19.0). Continuous data were expressed by means and standard deviations (SDs) for normal distribution, and median (range) for non-normal distribution. Categorical data were expressed in frequency and percentage. $\chi 2$ test and Wilcoxon's ranksum test were used to analyze statistical difference which $P$-value was set at less than 0.05 with statistical significance.

\section{Results}

\subsection{Characteristics of inpatient children}

Over the time of the study (2018.09-2019.08), HAdV was identified in 488 (6.9\%) specimens out of 7072 clinical inpatients with ARI at Children's Hospital of Zhejiang University School of Medicine in China. We retrieved 436 (89.3\%) samples from 436 cases in the clinical laboratory. Among 488 HAdV positive inpatients, 305 (62.5\%) were male, $183(37.5 \%)$ were female, the sex ratio was 1.68:1. The overall assay positive rate was $6.9 \%(488 / 7072)$. The assay positive rates were $7.04 \%(305 / 4330)$ and $6.7 \%$ $(183 / 2742)$ in male and female subjects respectively. No significant gender difference was observed $\left(c^{2}=0.358, p=0.550\right)$. The ages of the pediatric inpatients ranged from 39 days to 12 years old. HAdV infected inpatients' median age was 3.6 years (2.2 to 5.1 years). The assay positive rates of HAdV among age groups were significantly different $\left(c^{2}=31.691, p=0.000\right)$. The vast majority of HAdV-positive inpatients were between 6 months and $\leq 5$ years old $(393,80.5 \%)$. Subjects between 6 months and $\leq 2$ years old had the highest proportion of HAdV detection. The lowest HAdV-positive detection rate was observed in age $1-6$ months $(3.0 \%, 6 / 197)$, while subjects between 6 months and $\leq 2$ years old had the highest HAdV detection proportion $(8.7 \%, 123 / 1408)$ (Table 1).

\subsection{Clinical diagnosis and Clinical Characteristics of the HAdV-positive inpatients.}

As Table 2 shows, in these 488 inpatients enrolled with HAdV-positive respiratory infection, 111 (22.7\%) had an upper respiratory infection, $377(77.3 \%$ ) had lower respiratory tract infection (bronchitis and pneumonia). Among 343 (70.3\%) cases with pneumonia, 86 severe pneumonia (25.0\%) included. Fever $(456,93.4 \%)$ and cough $(462,94.7 \%)$ are the most common symptoms in the HAdV-positive patients, while the other clinical presentations were wheezing $(128,26.2 \%)$, shortness of breath $(72,14.8 \%)$, and extrapulmonary symptoms (Table1). The extrapulmonary symptoms included hepatic dysfunction, cardiac dysfunction, coagulation abnormalities, toxic encephalopathy. Hyperpyrexia $\left(\geq 39^{\circ} \mathrm{C}\right)$ accounts for $93.9 \%$ (439 cases). The length of fever time ranged from 5 to 17 days and the average time was 8.8 days. The great majority of inpatients' (373 cases, $76.2 \%$ ) fever time were more than 1 week. A total of $16 \%$ (78 cases) of the children had an underlying condition (with congenital heart diseases, and surgical diseases the most common condition). The stay of hospital ranged from 1 to 41 days, the median stay of hospital was 5.6 days (3 to 7 days). 55 children (11.3\%) required intensive care, and 2 ( $0.4 \%)$ died (Table 2). 


\subsection{Complication of the HAdV-positive inpatients}

Of 488 HAdV-positive cases, 40 cases presented acute respiratory insufficiency, of which, 21 cases with respiratory failure. 138 cases occurred extrapulmonary symptoms (including hepatic injury $(6.4 \%$, $31 / 488)$; marrow suppression $(5.3 \%, 26 / 488)$; myocardial injury $(3.5 \%, 17 / 488)$ and 2 cases with laryngemphraxis, cerebral infarction, and coagulation abnormalities respectively (Table 4). 368 cases had taken imageological examination (such as chest radiography, chest CT scan, and ultrasound). Intrapulmonary complications and intrathoracic complications were displayed in Table 4.

\subsection{Coinfection with other respiratory viruses}

Of 488 HAdV-positive inpatients, 213 comprised co-infection with other respiratory pathogens (including $191(89.7 \%, 191 / 213)$ with one other pathogen, $22(10.3 \%, 22 / 213)$ with two other pathogens). As shown in Table 3, influenza virus $(24.4 \%, 52 / 213)$, parainfluenza virus $(18.8 \%, 40 / 213) \rrbracket h u m a n$ respiratory syncytial virus $(13.6 \%, 29 / 213)$ were the most three frequently identified viruses in co-infected cases. The proportions of hyperpyrexia, duration of fever $>10$ days, severe pneumonia, and proportion of wheezing in the co-infection group were remarkably higher than those in HAdV single-infection group

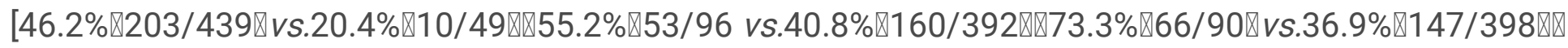

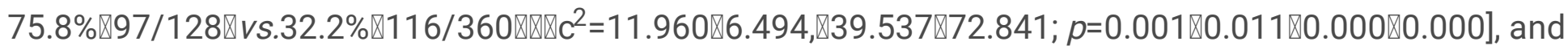

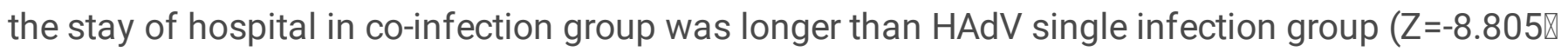
$p=0.000$ ) (Table 3). There was no significant difference between the single-infection group and the coinfection group in every age group $\square c^{2}=1.497, p=0.683$ ).

\subsection{HAdV species and type Distribution}

In the study period, we performed hexon gene amplification and Sanger sequencing on 162 HAdV-positive samples which were chosed radomly for further molecular type analysis. 155 (95.7\%) were successfully confirmed for the HAdV genotypes. They included species B (types $3,7,21,55$ ), species C (types 2, 5), species D (type 53), species E (4) (Fig 2A). Species B was the most common genotype [HAdV-B3, 87 (56.1\%); HAdV-B7, 48 (31.0\%); HAdV-B55, 4 (2.6\%); HAdV-B21, 1 (0.6\%)], followed by Species C [HAdV-C2, 5 (3.2\%), HAdV-C5, 2 (1.3\%)], Species D (2,1.3\%) and Species E (6,3.9\%). HAdV-B3 and HAdV-B7 were the most predominant types (Fig 2B). In this study, HAdV circulated all year round. HAdV-positive cases with RI mainly appeared in winter and summer, the HAdV detection rate peaked at $14.1 \%$ in January 2019(Fig.2C). Besides, HAdV-B3 can cause febrile respiratory infection in all age groups (Fig.2D). The HAdV genotype distribution in different severity groups showed that HAdV-B3 and HAdV-B7 occurred commonly in both groups, of which, HAdV-B55 occurred only in the severe group (Fig.2E). Two cases of HADV-B55 died in this study. HAdV-B7 positive detection rate in the severe group was significantly higher than the non-severe infected group $\left(X^{2}=95.857, p=0.000\right)$. The two dead inpatients genotype were HAdVB7 and HAdV-B55 respectively.

\section{Discussion}


HAdV was known as the most frequent cause of ARI in children which is a highly contagious pathogen. It is reported that the detection rate of HADV infection varied in different months, seasons, ages and regions, and circulated all year round, with epidemic outbreaks and clusters of severe cases occurring in individual regions ${ }^{[4,16,17]}$. Some HAdV types are common causes of severe respiratory tract infection in children. The majority of adenovirus respiratory infections are light to moderate and self-limited; however, sometimes they may cause life-threatening conditions, comorbidities, and serious sequela $\mathrm{e}^{[18-20]}$. The prognosis of severe HAdV infection in pediatric patients is poor, and many sequelae may be left, such as, bronchiectasis, interstitial fibrosis, etc., which can be life-threatening in severe cases ${ }^{[4,7]}$. Edmond $\mathrm{K}$ reported that association between adenovirus infection and a higher risk (55\%) for long-term sequelae compared to other causes of pneumonia in a meta-analysis of pneumonia in children younger than 5 years $^{[18]}$.

The present study retrospectively analyzed the epidemiology, clinical features, and genotype of 488 hospitalized children with adenovirus positive for acute respiratory tract infection in our hospital from September 2018 to August 2019. We reported the clinical features, the recent molecular epidemiological detected results of circulated HAdV strains in hospitalized children with RI in Zhejiang China. Our study showed the overall detection rate was $6.9 \%$ in HAdV-positive hospitalized children with ARIs and highlighted that the peak detection rate was in children between 6 months and $\leq 2$ years old; and suggesting that young children may be sensitive to HAdV in particular. Due to the protection of maternal antibodies, the detection rate was only $1.2 \%$ in HAdV-positive cases less than 6 months. We noted that $81.7 \%$ of $488 \mathrm{HAdV}$-positive cases were under 5 years which was consistent with other studies ${ }^{[21]}$. Our results were consistent with previous studies ${ }^{[21,22]}$. Pereira reported that children over 5 years old may have strong immunity to adenovirus infection ${ }^{[23]}$. December and January, February, July in the following year were discovered to be a high-detection month of HAdV respiratory infection, suggesting seasonality in HAdV respiratory infection. These results were consistent with Guangzhou reports in $2012{ }^{[24]}$. The season of high detection of respiratory adenovirus is different in different regions due to different climates which showed HAdV infection are associated with climatic factors (temperature and sunshine duration).

The clinical presentation of HAdV-positive ARIs is associated with age, genotype, and host immune function status. Previous studies have shown that age $<5$ years, underlying diseases, immunocompromised status were more likely to be infected and developed severe HAdV-positive infection ${ }^{[25]}$. We noted that fever and cough (presented by $93.4 \%$ and $94.7 \%$ ) were the most frequent symptoms observed in HAdV-positive hospitalized cases with ARIs, and the majority of children were less than 5 years old which is similar to the Italian study ${ }^{[26]}$. Wheezing and shortness of breath were more frequent in the severe group. Patients with severe HAdV infections have prolonged fever and required longer hospitalization. Of 488 hospitalized HAdV-positive cases, we noted that lower respiratory tract infections $(70.3 \%$ in pneumonia and $7.0 \%$ in bronchitis), upper respiratory tract infection were found in $77.3 \%$, death occurred in $0.4 \%$ of cases. Of the 343 cases of pneumonia, $17.6 \%$ (86/488) were serious. It is different in severity in HAdV infection under the age, environmental status, and immunological status 
of the patient. It is hard to clinical diagnose HAdV-positive ARI from other pathogens infections (such as mycoplasma pneumonia, influenza, etc.) because the clinical presentation seemed to be alike. Therefore, we require further study on coinfections with HAdV and other respiratory pathogens.

Many studies have reported co-infection with other respiratory pathogens. In our study, the co-infections rate was $43.6 \%(213 / 488)$ that was in accord with previous reports ${ }^{[27]}$. Co-infection with other respiratory

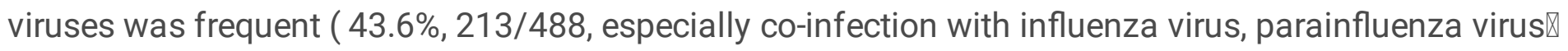
human respiratory syncytial virus) in the study. And 39.1\% (191/ 488) were double infected, $4.5 \%$ $(22 / 488)$ were multiply infected. Previous clinical research reported that co-infected patients generally have a prolonged fever time, severe pneumonia, wheezing, and duration of hospitalization which was consistent with our study.

According to previous studies, HAdV- $B(3,7,21), \operatorname{HAdV-C}(1,2,5,6)$ and HAdV-E (4)were the most frequent HAdV species which caused ARIs in children worldwide ${ }^{[28]}$. HAdV-3 and HAdV-7 were the most frequent genotypes in China ${ }^{[29]}$. HAdV-7 is closely related to severe pneumonia and high mortality compared with other genotypes in infants and children ${ }^{[30,31]}$. Although patients from every age group may be susceptible to HAdV infections, more cases of HAdV infections were observed among less than 4 years old children -in this study. HAdV-B $(90.3 \%, 140 / 155)$ was the most commonly identified genotype compared with other HAdV types, suggesting that young children with ARIs may be particularly susceptible to species B. HAdV-B3(56.1\%, 87/155) was the dominant genotype in cases with HAdV respiratory infection. Over $60 \%$ of severe HAdV pneumonia tend to attribute to HAdV-B7. It also shows that the severity of HAdV pneumonia is related to HAdV genotypes and it is important to identify HAdV genotypes to correlate clinical disease and symptoms with all genotypes.

A nationwide epidemiological surveillance program for HAdV infection has not yet been built in China currently. HAdV identification is usually conducted for laboratory research and it is hard to be applied to the routine clinical diagnosis. We need long-time surveillance and rapid diagnostic methods of HAdV infection. Rapid detection is important to avoid unnecessary antibiotic consumption for HAdV respiratory infections.

This research had certain limitations. Firstly, this is a one-year single-center study and these results didn't generalize to other children across China; Secondly, we were unable to proceed with comparative analyses between genotypes owing to small sample size; thirdly, due to the retrospective analysis, we were unable to record all the symptoms and signs which may cause exposure misclassification.

\section{Conclusion}

HAdV detection rate is related to age and season. Bronchopneumonia accounts for about $70 \%$ HAdV positive inpatients. The common manifestations include hyperpyrexia, cough, wheezing, and shortness of breath. HAdV-B3 and HAdV-B7 are the most common types in children diagnosed with RI. 


\section{Abbreviations}

HAdV: Human adenovirus ; RI: Respiratory infection ; NPAs: nasopharyngeal aspirates; DFA囚Direct Immunofluorescence Assay.

\section{Declarations}

\section{Acknowledgements}

Not applicable.

\section{Authors' contributions}

WC, LJ, MY, CJ and BJ analyzed and interpreted the patient data. WC was a major contributor who performed the statistical analysis, drafted and reviewed the manuscript. CY was responsible for research design. All authors read and approved the final manuscript.

\section{Funding}

Supported by the Medical and Health Science and Technology Project of Zhejiang Province邓2018KY451区.

\section{Availbility of data and materials}

Not applicable.

\section{Ethics approval and consent to participate}

The study was approved by the Medical Ethics Committee of Children's Hospital Affiliated to Zhejiang University Medical College (2019-IRB 063).

\section{Consent for publication}

Written informed consent for publication was obtained from all participants and their parents.

\section{Competing interests}

The authors declare that they have no competing interests.

\section{Author details:}

${ }^{1}$ Department of Infectious Disease, Children's Hospital of Zhejiang University School of Medicine, National Clinical Research Center for Child Health, National Children's Regional Medical Center, 3333 Binsheng Road,310052, Hangzhou, Zhejiang province, China

${ }^{2}$ Department of Otolaryngology-Head and Neck Surgery, Children's Hospital of Zhejiang University School of Medicine, National Clinical Research Center for Child Health, National Children's Regional Medical 
Center, 3333 Binsheng Road,310052, Hangzhou, Zhejiang province, China

\section{References}

1. Lion T. Adenovirus infections in immunocompetent and immunocompromised patients[J]. Clin Microbiol Rev, 2014, 27(3): 441-462.10.1128/cmr.00116 - 13.

2. Ismail AM, Lee JS, Lee JY, et al. Adenoviromics: Mining the Human Adenovirus Species D Genome[J]. Front Microbiol, 2018, 9: 2178.10.3389/fmicb.2018.02178.

3. Finianos M, Issa R, Curran MD, et al. Etiology, seasonality, and clinical characterization of viral respiratory infections among hospitalized children in Beirut, Lebanon[J]. J Med Virol, 2016, 88(11): 1874-1881.10.1002/jmv.24544.

4. Sandkovsky U, Vargas L,Florescu DF. Adenovirus: current epidemiology and emerging approaches to prevention and treatment[J]. Curr Infect Dis Rep, 2014, 16(8): 416.10.1007/s11908-014-0416-y.

5. Jain S, Williams DJ, Arnold SR, et al. Community-acquired pneumonia requiring hospitalization among U.S. children[J]. N Engl J Med, 2015, 372(9): 835-845.10.1056/NEJMoa1405870.

6. Xie L, Zhang B, Xiao N, et al. Epidemiology of human adenovirus infection in children hospitalized with lower respiratory tract infections in Hunan, China[J]. J Med Virol, 2019, 91(3): 392400.10.1002/jmv.25333.

7. Lynch JP 3rd,Kajon AE. Adenovirus: Epidemiology, Global Spread of Novel Serotypes, and Advances in Treatment and Prevention[J]. Semin Respir Crit Care Med, 2016, 37(4): 586-602.10.1055/s-00361584923.

8. Zhang SY, Luo YP, Huang DD, et al. Fatal pneumonia cases caused by human adenovirus 55 in immunocompetent adults[J]. Infect Dis (Lond), 2016, 48(1): 40-

47.10.3109/23744235.2015.1055585.

9. Tórtora RP, Guimarães $M$, de Souza LM, et al. Adenovirus species $C$ detection in children under four years of age with acute bronchiolitis or recurrent wheezing[J]. J Clin Virol, 2015, 73: 7780.10.1016/j.jcv.2015.11.007.

10. Yu P, Ma C, Nawaz M, et al. Outbreak of acute respiratory disease caused by human adenovirus type 7 in a military training camp in Shaanxi, China[J]. Microbiol Immunol, 2013, 57(8): 553560.10.1111/1348-0421.12074.

11. Lin YC, Lu PL, Lin KH, et al. Molecular Epidemiology and Phylogenetic Analysis of Human Adenovirus Caused an Outbreak in Taiwan during 2011[J]. PLoS One, 2015, 10(5): e0127377.10.1371/journal.pone.0127377.

12. Ko JH, Woo HT, Oh HS, et al. Ongoing outbreak of human adenovirus-associated acute respiratory illness in the Republic of Korea military, 2013 to 2018[J]. Korean J Intern Med, 2021, 36(1): 205213.10.3904/kjim.2019.092.

13. Hage E, Huzly D, Ganzenmueller T, et al. A human adenovirus species B subtype 21 a associated with severe pneumonia[J]. J Infect, 2014, 69(5): 490-499.10.1016/j.jinf.2014.06.015. 
14. Houng HS, Gong H, Kajon AE, et al. Genome sequences of human adenovirus 14 isolates from mild respiratory cases and a fatal pneumonia, isolated during 2006-2007 epidemics in North America[J]. Respir Res, 2010, 11(1): 116.10.1186/1465-9921-11-116.

15. Bradley JS, Byington CL, Shah SS, et al. The management of community-acquired pneumonia in infants and children older than 3 months of age: clinical practice guidelines by the Pediatric Infectious Diseases Society and the Infectious Diseases Society of America[J]. Clin Infect Dis, 2011, 53(7): e25-76.10.1093/cid/cir531.

16. Wang W, Liu Y, Zhou Y, et al. Whole-genome analyses of human adenovirus type 55 emerged in Tibet, Sichuan and Yunnan in China, in 2016[J]. PLoS One, 2017, 12(12): e0189625.10.1371/journal.pone.0189625.

17. Yu Z, Zeng Z, Zhang J, et al. Fatal Community-acquired Pneumonia in Children Caused by Reemergent Human Adenovirus 7d Associated with Higher Severity of IIIness and Fatality Rate[J]. Sci Rep, 2016, 6: 37216.10.1038/srep37216.

18. Edmond K, Scott S, Korczak V, et al. Long term sequelae from childhood pneumonia; systematic review and meta-analysis[J]. PLoS One, 2012, 7(2): e31239.10.1371/journal.pone.0031239.

19. Larrañaga $C$, Kajon A, Villagra $E$, et al. Adenovirus surveillance on children hospitalized for acute lower respiratory infections in Chile (1988-1996)[J]. J Med Virol. 2000;60(3):342-6.

20. Carballal G, Videla C, Misirlian A, et al. Adenovirus type 7 associated with severe and fatal acute lower respiratory infections in Argentine children[J]. BMC Pediatr, 2002, 2: 6.10.1186/1471-2431-2-6.

21. Kwon HJ, Rhie YJ, Seo WH, et al. Clinical manifestations of respiratory adenoviral infection among hospitalized children in Korea[J]. Pediatr Int, 2013, 55(4): 450-454.10.1111/ped.12108.

22. Wu $P Q$, Zeng SQ, Yin GQ, et al. Clinical manifestations and risk factors of adenovirus respiratory infection in hospitalized children in Guangzhou, China during the 2011-2014 period[J]. Medicine (Baltimore), 2020, 99(4): e18584.10.1097/md.0000000000018584.

23. Pereira MS. Adenovirus infections[J]. Postgrad Med J, 1973, 49(577): 798801.10.1136/pgmj.49.577.798.

24. Zou L, Zhou J, Li H, et al. Human adenovirus infection in children with acute respiratory tract disease in Guangzhou, China[J]. Apmis, 2012, 120(8): 683-688.10.1111/j.1600-0463.2012.02890.x.

25. Gray GC, McCarthy T, Lebeck MG, et al. Genotype prevalence and risk factors for severe clinical adenovirus infection, United States 2004-2006[J]. Clin Infect Dis, 2007, 45(9): 11201131.10.1086/522188.

26. Esposito S, Zampiero A, Bianchini S, et al. Epidemiology and Clinical Characteristics of Respiratory Infections Due to Adenovirus in Children Living in Milan, Italy, during 2013 and 2014[J]. PLoS One, 2016, 11(4): e0152375.10.1371/journal.pone.0152375.

27. Probst V, Datyner EK, Haddadin Z, et al. Human adenovirus species in children with acute respiratory illnesses[J]. J Clin Virol, 2021, 134: 104716.10.1016/j.jcv.2020.104716.

28. Shimada $Y$, Ariga T, Tagawa $Y$, et al. Molecular diagnosis of human adenoviruses $d$ and e by a phylogeny-based classification method using a partial hexon sequence[J]. J Clin Microbiol, 2004, 
42(4): 1577-1584.10.1128/jcm.42.4.1577-1584.2004.

29. Liu C, Xiao Y, Zhang J, et al. Adenovirus infection in children with acute lower respiratory tract infections in Beijing, China, 2007 to 2012[J]. BMC Infect Dis, 2015, 15: 408.10.1186/s12879-015$1126-2$.

30. Li QG, Zheng QJ, Liu YH, et al. Molecular epidemiology of adenovirus types 3 and 7 isolated from children with pneumonia in Beijing[J]. J Med Virol, 1996, 49(3): 170-177.10.1002/(sici)10969071(199607)49:3 < 170::Aid-jmv3 > 3.0.Co;2 - 1

31. Lai CY, Lee CJ, Lu CY, et al. Adenovirus serotype 3 and 7 infection with acute respiratory failure in children in Taiwan, 2010-2011[J]. PLoS One, 2013, 8(1): e53614.10.1371/journal.pone.0053614.

\section{Tables}

Table 1 HAdV-positive respiratory infection in children of different ages and gender with ARTIs

\begin{tabular}{|lllll|}
\hline Characteristics & Number of children & Number of HAdV-positive children $\mathbf{n}(\%)$ & $c^{2}$ & P-value \\
\hline Ages (years) & & & 31.691 & 0.000 \\
$<0.5$ & 197 & $6(3.0)$ & & \\
$\geq 0.5$ to $₫ 2$ & 1408 & $123(8.7)$ & & \\
$\geq 2$ to $\llbracket 5$ & 3519 & $270(7.7)$ & & \\
$\geq 5$ & 1948 & $89(4.6)$ & & \\
Gender & & & & \\
Male & 2742 & $305(7.04)$ & & \\
Female & 4330 & $183(6.70)$ & & \\
total & 7072 & $488(6.90)$ & & \\
\hline
\end{tabular}

Table 2 Demographic characteristics and clinical features of HAdV-positive respiratory infection in hospitalized children 


\begin{tabular}{|c|c|c|}
\hline Variables & Clinical Characteristics & $\begin{array}{l}\text { Number of HAdV-positive children } n \\
\text { (\%) }\end{array}$ \\
\hline \multirow{13}{*}{$\begin{array}{l}\text { Clinical } \\
\text { manifestation }\end{array}$} & Fever & 456ه93.4】 \\
\hline & 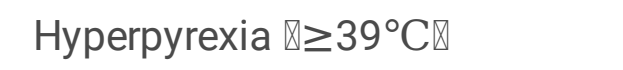 & $439 \otimes 90.0 \rrbracket$ \\
\hline & Duration of fever> 1 week & $373 \rrbracket 76.4 \rrbracket$ \\
\hline & Duration of fever $>10$ days & 96ه19.7》 \\
\hline & Cough & $462 \rrbracket 94.7 \rrbracket$ \\
\hline & Wheezing & $128 \rrbracket 26.2 \rrbracket$ \\
\hline & Somnolence & $87 \rrbracket 17.8 \rrbracket$ \\
\hline & Shortness of breath & $72 \bigotimes 14.8 \rrbracket$ \\
\hline & Pertussoid & $9 \rrbracket 1.8 \rrbracket$ \\
\hline & Vomiting and Diarrhea & $37 \rrbracket 7.6 \rrbracket$ \\
\hline & $\begin{array}{l}\text { Convulsion and altered mental } \\
\text { status }\end{array}$ & $31 \rrbracket 6.4 \rrbracket$ \\
\hline & Bulbar conjunctival congestion & $10 \otimes<. / \otimes$ \\
\hline & Urinary irritation symptoms & \\
\hline \multirow[t]{4}{*}{ Laboratory test } & High WBC count & LOAU. $1 \Delta$ \\
\hline & Decreased WBC count & $11 \otimes<.3 \otimes$ \\
\hline & High CRP\>8mg/L】 & 40囚8.2】 \\
\hline & High PCT $\otimes>0.5 \mathrm{ng} / \mathrm{ml} \otimes$ & $17 \otimes 2.3 \otimes$ \\
\hline \multirow[t]{6}{*}{ Underlying diseases } & Congenital heart diseases & $10 \otimes 3.3 \otimes$ \\
\hline & Surgical diseases & $13 \otimes 2.7 凶$ \\
\hline & Malnutrition & 12ख2.5区 \\
\hline & Arrhythmia & 10冈2.1区 \\
\hline & Epilepsy & 5囚1.0区 \\
\hline & Asthma & $3 \otimes 0.6 \rrbracket$ \\
\hline \multirow{2}{*}{ Sequelae } & Dronchingtario & $3 \varangle 0.6 \rrbracket$ \\
\hline & Bronchiolitis Obliterans & $3 \otimes 0.6 \rrbracket$ \\
\hline
\end{tabular}

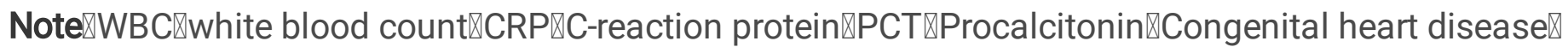
including atrial septal defect】ventricular septal defect!patent ductus arteriosus $\square$ Aortic and tricuspid valve 
disease $₫$ Surgical Diseases including crissum abscess $\square$ hiatal hernia $\square$ indirect inguinal hernia $\square$ congenital

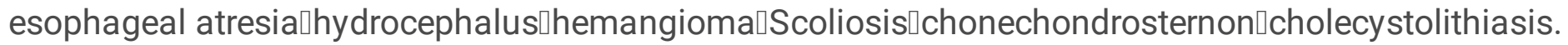

Table 3 Comparison of clinical characteristics of children with HAdV co-infection and single infection [n (\%)]

\begin{tabular}{|c|c|c|c|c|}
\hline Characteristics & Single-infection & Co-infection & $c 2 / z$ & P-value \\
\hline Ages(years) & & & 1.497 & 0.683 \\
\hline$\otimes 0.5$ & $4 \rrbracket 1.5 \rrbracket$ & $2 \varangle 0.9 \rrbracket$ & & \\
\hline$\geq 0.5$ to 2 & $73 \llbracket 26.5 \rrbracket$ & $50 \bowtie 23.5 \rrbracket$ & & \\
\hline$\geq 2$ to 5 & 152邓55.3邓 & $118 \rrbracket 55.4 \rrbracket$ & & \\
\hline$\geq 5$ & $46 \rrbracket 16.7 \rrbracket$ & $43 \rrbracket 20.2 \rrbracket$ & & \\
\hline Gender (Male/Female, n) & $175 / 100$ & $130 / 83$ & 0.347 & 0.556 \\
\hline 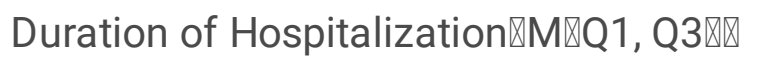 & $4.0 \rrbracket 3.0,5.0 \rrbracket$ & $6 \rrbracket 4.0,9.0 \rrbracket$ & -8.805 & 0.000 \\
\hline Fever & $247 \rrbracket 89.8 \rrbracket$ & 209₫98.1区 & 13.508 & 0.000 \\
\hline Hyperpyrexia $\triangle \geq 39^{\circ} \mathrm{C} \otimes$ & $236 \rrbracket 85.8 \rrbracket$ & 203凶95.3凶 & 11.960 & 0.001 \\
\hline Duration of Fever $\otimes 10 d$ & $43 \otimes 15.6 \rrbracket$ & $53 \rrbracket 24.9 \rrbracket$ & 6.494 & 0.011 \\
\hline Wheezing & $31 \otimes 11.3 \rrbracket$ & $97 \rrbracket 45.5 \rrbracket$ & 72.841 & 0.000 \\
\hline Underlying Diseases & $37 \rrbracket 13.5 \rrbracket$ & 41囚19.2】 & 3.001 & 0.083 \\
\hline High WBC count & $23 \llbracket 8.4 \rrbracket$ & $63 \rrbracket 29.6 \rrbracket$ & 37.209 & 0.000 \\
\hline \multirow[t]{2}{*}{ High CRP\>8mg/L】 } & $12 \rrbracket 40.0 \rrbracket$ & $16 \llbracket 30.2 \rrbracket$ & 0.825 & 0.364 \\
\hline & 13ه43.3区 & $27 \nabla 50.9 \rrbracket$ & 0.444 & 0.505 \\
\hline
\end{tabular}

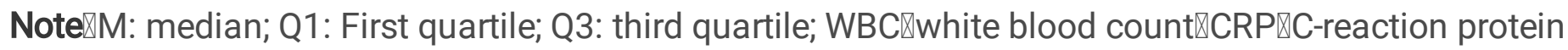
Table 4 Complication of HAdV-positive respiratory infection in children 


\begin{tabular}{|c|c|c|}
\hline Variables & Complications & $\begin{array}{l}\text { Number of HAdV-positive } \\
\text { children } n(\%)\end{array}$ \\
\hline \multirow{9}{*}{$\begin{array}{l}\text { Intrapulmonary and intrathoracic } \\
\text { complications }\end{array}$} & \multirow{4}{*}{$\begin{array}{l}\text { pulmonary } \\
\text { consolidation } \\
\text { pulmonary emphysema } \\
\text { pulmonary atelectasis }\end{array}$} & $108 \rrbracket 29.3 \rrbracket$ \\
\hline & & 86『23.4】 \\
\hline & & $56 \rrbracket 15.2 \rrbracket$ \\
\hline & & $57 \rrbracket 15.4 \rrbracket$ \\
\hline & serous etrusion & $56 \rrbracket 15.2 \rrbracket$ \\
\hline & pleural etrusion & $40 \rrbracket 8.2 \rrbracket$ \\
\hline & $\begin{array}{l}\text { acute respiratory } \\
\text { insufficiency }\end{array}$ & $21 \rrbracket 4.3 \rrbracket$ \\
\hline & respiratory failure & $8 \rrbracket 2.17 \rrbracket$ \\
\hline & pericardial effusion & $1 \otimes 0.2 \rrbracket$ \\
\hline \multirow{10}{*}{ extrapulmonary symptoms } & necrotizing pneumonia & $31 \rrbracket 6.4 \rrbracket$ \\
\hline & hepatic injury & $26 \rrbracket 5.3 \rrbracket$ \\
\hline & marrow suppression & 17囚3.5区 \\
\hline & myocardial injury & $2 \bowtie 0.4 \rrbracket$ \\
\hline & $\begin{array}{l}\text { coagulation } \\
\text { abnormalities }\end{array}$ & $2 \varangle 0.4 \rrbracket$ \\
\hline & corohral infarction & $2 \bowtie 0.4 \rrbracket$ \\
\hline & 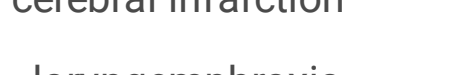 & $2 \varangle 0.4 \rrbracket$ \\
\hline & 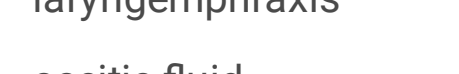 & $2 \bowtie 0.4 \rrbracket$ \\
\hline & pelvic effusion & $1 \otimes 0.2 \rrbracket$ \\
\hline & $\begin{array}{l}\text { hemophagocytic } \\
\text { syndrome }\end{array}$ & \\
\hline
\end{tabular}

\section{Figures}




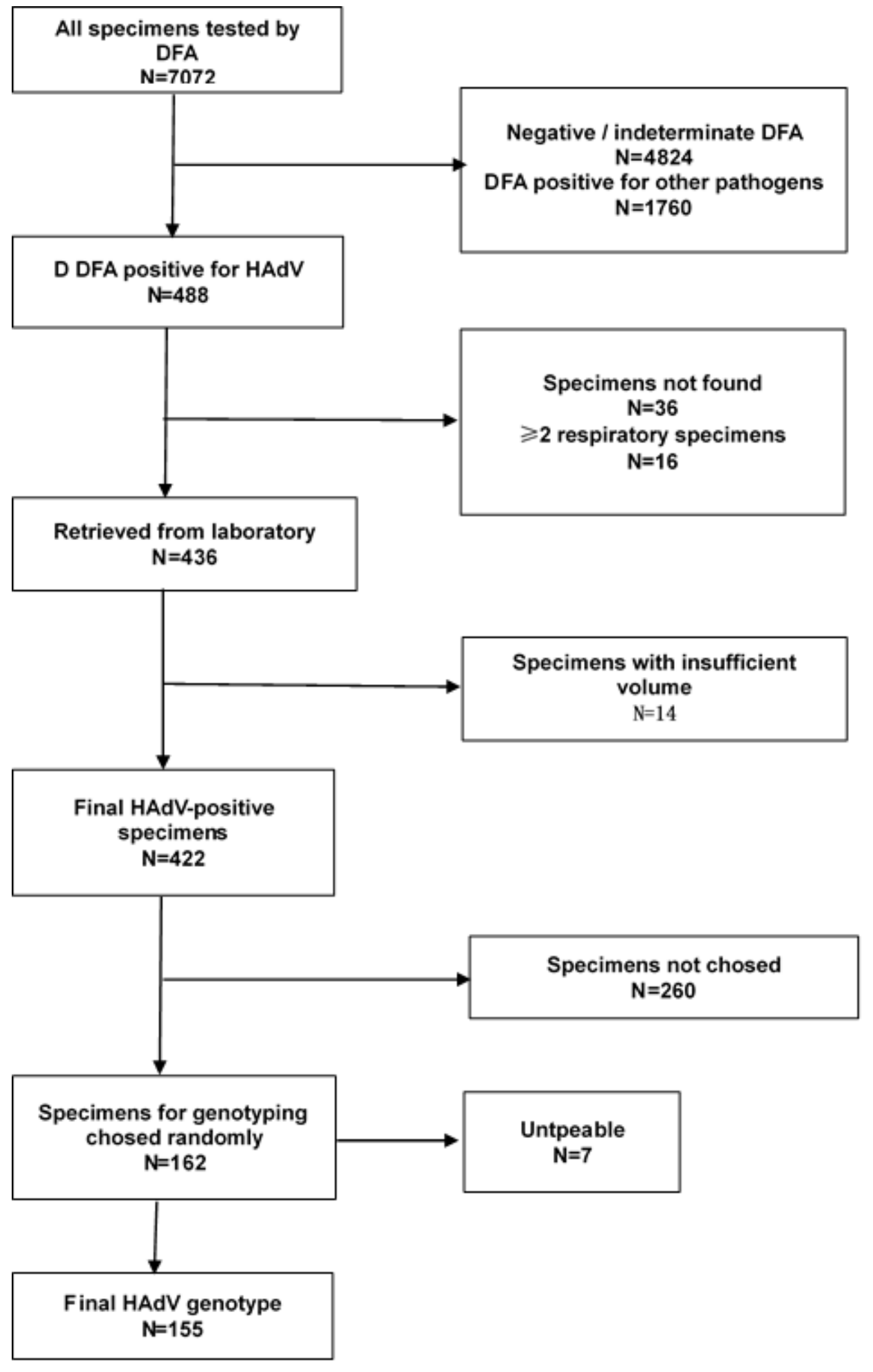

\section{Figure 1}

Study Inclusion Algorithm and Result of Respiratory Specimens Testing Abbreviation: DFA, direct immunofluorescence assay; HAdV, human adenovirus; 

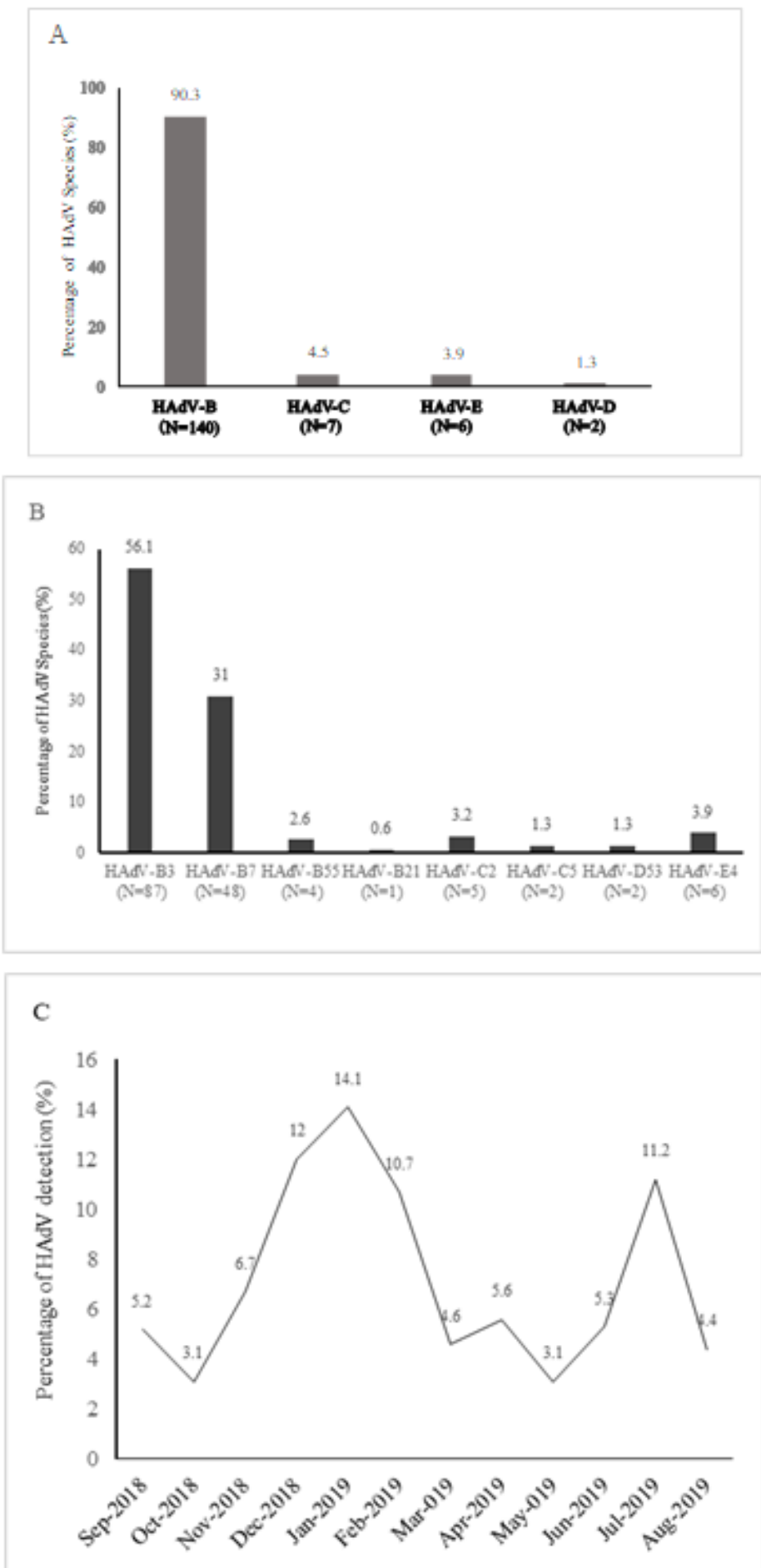

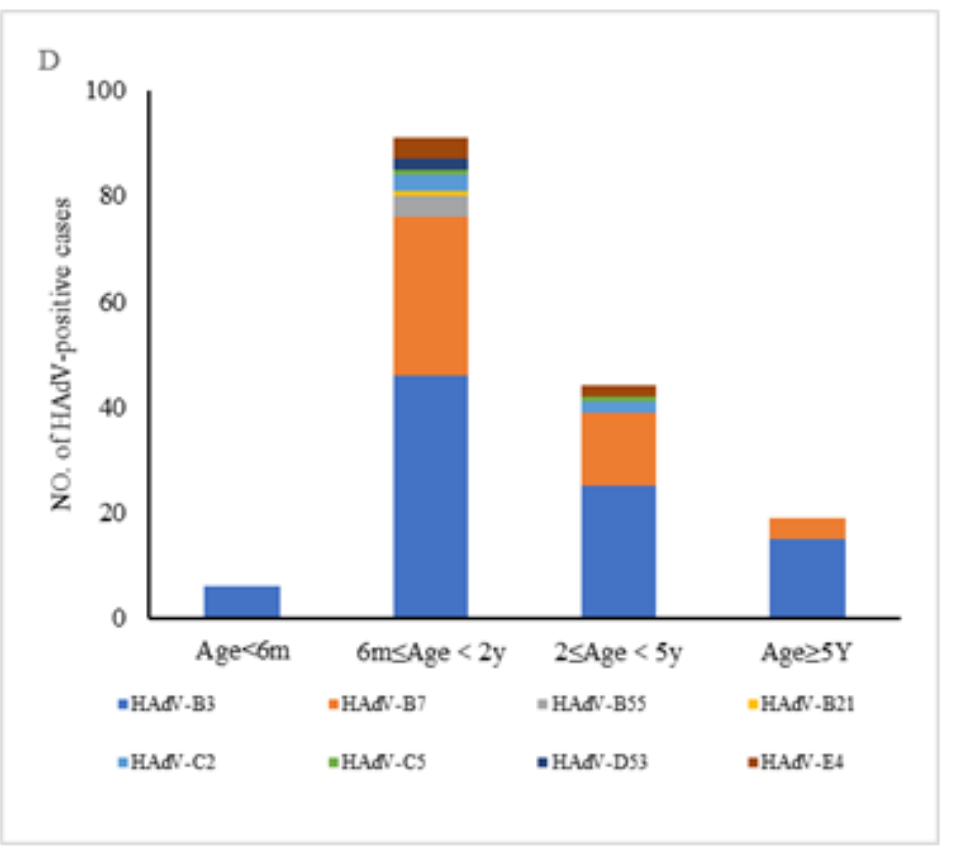

\section{E}

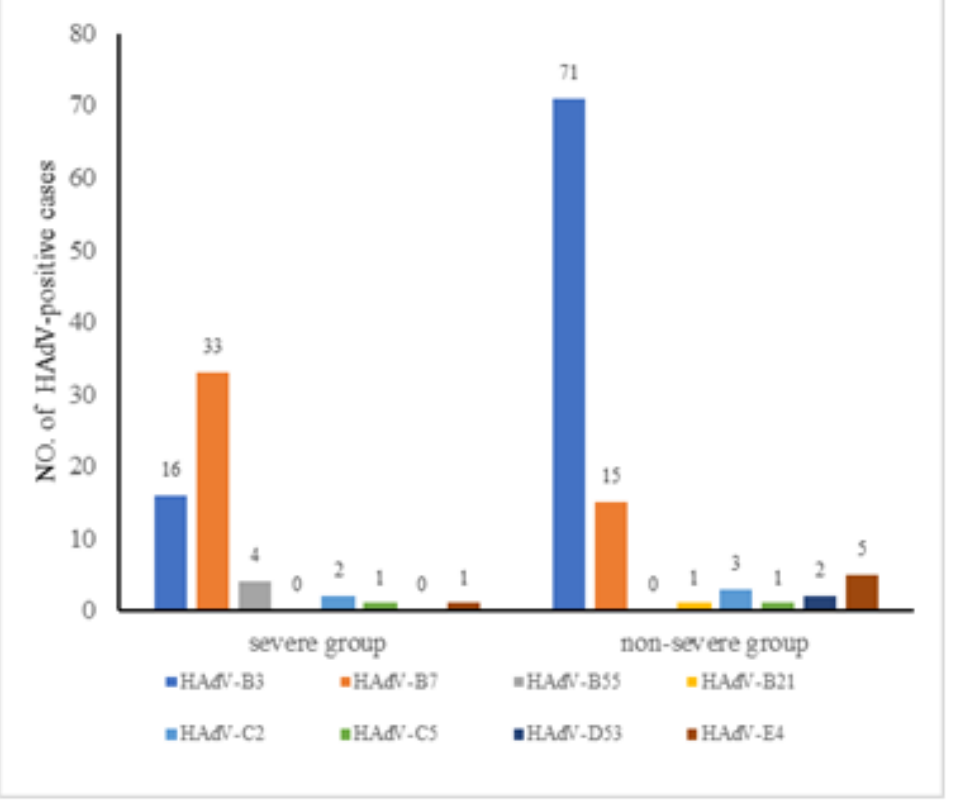

\section{Figure 2}

A. Distribution of HAdV Species of Enrolled Inpatient with ARTI; B. Distribution of HAdV Types of Enrolled Inpatient with ARTI; C. HAdV-positive detection rate monthly; D. Distribution of HAdV Types of Inpatient with ARTI in Different Age Group; E. Distribution of HAdV Types of Inpatient with ARTI in Different severity of Disease Group; 